\title{
Mathematical Modelling of a Specialized Vehicle Caterpillar Mover Dynamic Processes Under Condition of the Distributing the Parameters of the Caterpillar
}

\author{
Serhii Strutynskyi $^{1 *}$, Volodymyr Kravchuk ${ }^{2}$, Roman Semenchuk ${ }^{1}$. \\ ${ }^{I}$ National Technical University of Ukraine «Igor Sikorsky Kyiv Polytechnic Institute» \\ ${ }^{2}$ Leonid Pogorilly State Scientific Institution "Ukrainian Research Institute for forecasting and testing equipment and technologies for \\ agricultural production" \\ *Corresponding author E-mail: strutynskyi@gmail.com
}

\begin{abstract}
Features of a specialized vehicle caterpillar mover are considered. A dynamic model of caterpillar transverse oscillations as a system with distributed parameters is proposed. The differential equation in partial derivatives is obtained and its connection in the form of a decomposition by normal oscillation types is found. The analysis of frequencies and the type of caterpillar oscillations has been made. Inertial loads have been found. The mathematical model of forced oscillations of a caterpillar under the influence of random loads is developed and the mathematical modelling is executed. An analysis of the vibration movements and vibration velocities of the intersections of the caterpillar is carried out. The mathematical modeling of the caterpillar oscillations caused by stochastic discrete loads was carried out. A model of random discrete loads has been developed, calculations of the realization of random loads are performed. It was established that the transverse discrete loads occur in points of mass concentration on the caterpillar surface.
\end{abstract}

Keywords: caterpillar; frequencies; mathematical model; patterns; random oscillations; transient processes; vehicle

\section{Introduction}

Specialized vehicles are used in terrestrial robotic workstations. They are equipped with manipulators or special devices. Modern terrestrial workstations have a low movement speed up to 20 ... 30 $\mathrm{km} \backslash \mathrm{h}$. It reduces their effectiveness. Increasing the speed leads to intensive dynamic loads in a special vehicle chain (caterpillar) mover in particular. Reducing dynamic loads could be achieved by improving the caterpillar mover according to the results of special studies. Therefore, work in this direction is relevant.

The problem in general is the development of highly efficient special vehicles with a chain (caterpillar) mover. The problem is related to important scientific and practical tasks of great importance for the state security of Ukraine.

Recent studies and publications outline the results of the development and research of terrestrial robotic workstations for special purpose [1]. It is noted that robotic systems have small dimensions $(1 . .2 \mathrm{~m})$ and include a chassis and a working module, for example, a manipulator [2]. Mechanisms with parallel kinematic structures are often used as a manipulator [3]. These mechanisms have low materials consumption and broad functionality [4].

Specialized vehicles use wheel or caterpilar chassis. The latter are more effective for use. There are results of researches of geometrical and power parameters of a special vehicle caterpillar mover in literary sources $[5,6]$. Characteristics of the flotation ability, controllability and performance properties of the vehicles of this type are described [7, 8]. There are results of studying dynamic processes in caterpillar mover dynamic processes in literary sources [9].

The peculiarities of dynamics of drives, characteristics of transitional processes [10] in caterpillar movers are established. Dynam- ic properties of the caterpillar are investigated through numerical calculation by the finite element method [11]. The disadvantage of these studies is the specification of the calculations and, accordingly, the insignificant degree of the simulation results generalization.

It could be achieved by applying combined methods that include analytical models of the system in distributed parameters and numerical methods. Similar approaches to the research of dynamic processes in caterpillar movers under condition of their parameters distribution have not been found in literary sources. Previously undecided parts of the general problem include the study of the peculiarities of dynamic processes in the chain (caterpillar) movers in specialized vehicles using analytical models of caterpillars as systems with distributed parameters.

The purpose of the research described in this article is to establish the peculiarities of the dynamic processes caused by the distribution of parameters of a specialized vehicle chain (caterpillar) mover. The task of the research is the definition of the laws of the fundamental and forced oscillation processes in the caterpillar mover under condition of its parameters distribution along the length and random discrete caterpillar load.

\section{Description of Main Research Materi- al.Calculation of Parameters of Fundamental Transverse Oscillations of a Caterpillar as a System with Distributed Parameters}

Specialized vehicles are designed to work with non-secure objects and are operated remotely. They have a caterpillar mover chassis and a manipulator working module (Fig. 1). 

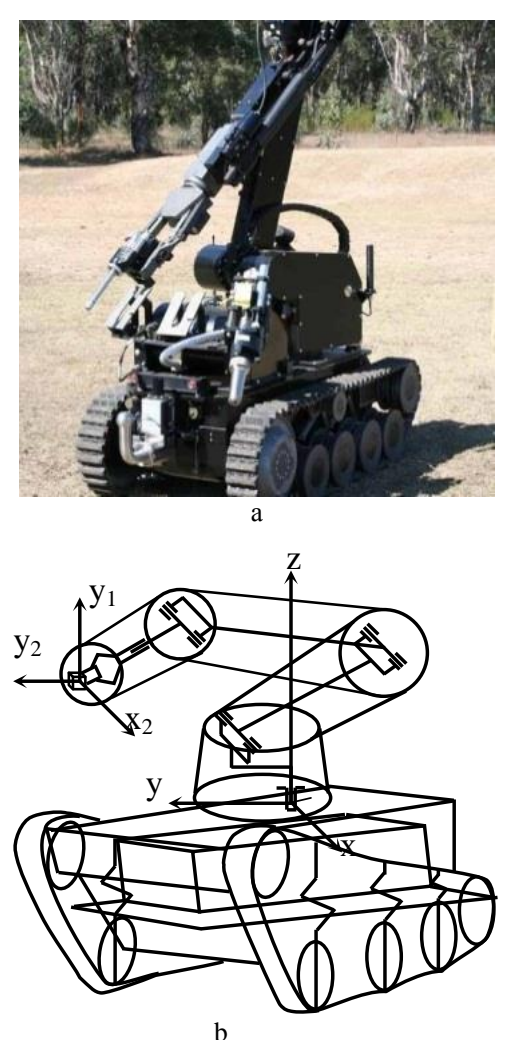

Fig. 1: General view (a) and schematic diagram of (b) a specialized vehicle caterpillar mover.

The caterpillar chains are made in the shape of circular chains that move by means of driving transmissions. Separate links of the caterpillar chain 1 are hingedly connected to each other and have sufficient flexibility for smooth enveloping driving 2 and driven by 3 reels of the caterpillar mover (Fig. 2).

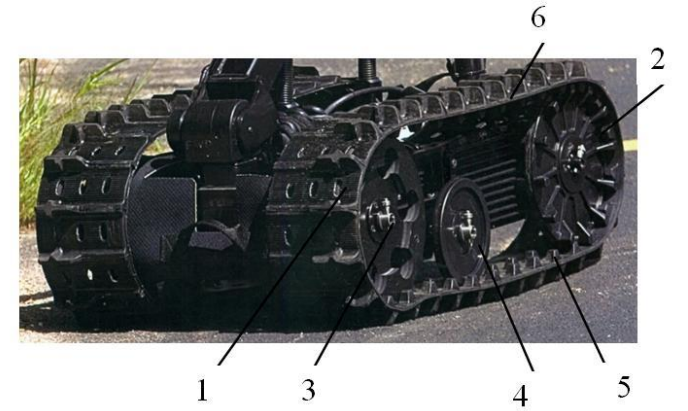

Fig. 2 The main components of a specialized vehicle caterpillar mover.

The caterpillar mover of the vehicle has one or more rollers 4 which press the lower part of the caterpillar 5 to the roadway. The upper part of the caterpillar 6 is held up by elastic forces in a tension. In some cases, additional support rollers are used.

In specialized vehicles different types of a caterpillar mover with different numbers and shape of chain links are used. Usually, the number of links is $30 \ldots 40$, and the driving rail has $12 \ldots 16$ teeth that come in contact with the holes of the caterpillar.

To define the peculiarities of stochastic dynamic processes in a caterpillar mover its mathematical model as a system with distributed parameters is designed [12]. Let's consider the top of the caterpillar. Transverse oscillations arise in it as a result of dynamic loads. Their calculation is carried out under condition of the distribution of parameters (mass) of the caterpillar in length (Fig. 3).

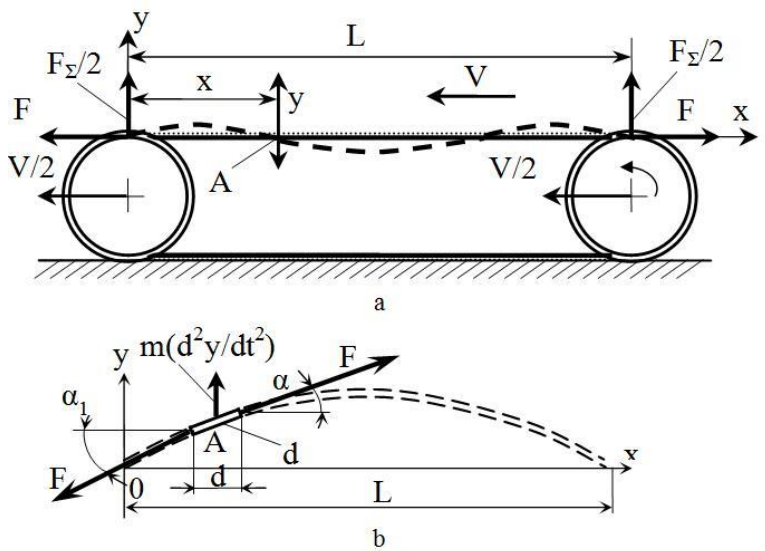

Fig. 3: Occurrence of transverse oscillations of a specialized vehicle caterpillar (a) and movement of the elementary section of the caterpillar with its transverse oscillations (b).

Determination of transverse oscillations of a caterpillar mover is carried out in Euler variables. Moving from full derivatives over time to local derivatives we get:

$$
\begin{aligned}
& \frac{\mathrm{dy}}{\mathrm{dt}}=\frac{\partial \mathrm{y}}{\partial \mathrm{t}}+\frac{\partial \mathrm{y}}{\partial \mathrm{x}} \cdot \frac{\partial \mathrm{x}}{\partial \mathrm{t}}=\frac{\partial \mathrm{y}}{\partial \mathrm{t}}+\frac{\partial \mathrm{y}}{\partial \mathrm{x}} \mathrm{V} \\
& \frac{\mathrm{d}^{2} \mathrm{y}}{\mathrm{dt}^{2}}=\frac{\partial^{2} \mathrm{y}}{\partial \mathrm{t}^{2}}+2 \mathrm{~V} \frac{\partial^{2} \mathrm{y}}{\partial \mathrm{x} \partial \mathrm{t}}+\mathrm{V}^{2} \frac{\partial^{2} \mathrm{y}}{\partial \mathrm{x}^{2}}
\end{aligned}
$$

where V - speed of the caterpillar movement; $y$ - is the transverse movement of the caterpillar at the point $\mathrm{A}$ with the coordinate $\mathrm{x}, \mathrm{t}$ time.

Let's consider the movement of the elementary section of the caterpillar dx- length (Fig. 3b). The inclination angle of the elementary section of the caterpillars relatively to the axis $\mathrm{x}$ will be determined through the derivative of the center line of the caterpillar. For the beginning of an elementary section $\mathrm{dx}$ in length we have:

$$
\alpha_{1}=\operatorname{arctg} \frac{\partial \mathrm{y}}{\partial \mathrm{x}} \text {. }
$$

For the end of the elementary section the inclination angle:

$$
\alpha_{2}=\operatorname{arctg}\left(\frac{\partial y}{\partial x}+\frac{\partial^{2} y}{\partial x^{2}} d x\right) \text {. }
$$

The equilibrium equation of the elementary section of the caterpillar is determined in the projections of forces on the axis in:

$$
F\left(\frac{\partial y}{\partial x}+\frac{\partial^{2} y}{\partial x^{2}} d x\right)-F \frac{\partial y}{\partial x}-m \frac{d^{2} y}{d^{2}}(d x)=0,
$$

where $\mathrm{F}$ is the tension strength of the caterpillar; $\mathrm{m}$ - linear mass of the caterpillar.

After substituting value of derivative into equilibrium equation (1) and conversions we obtain the equation of transverse oscillations of the caterpillar:

$$
\frac{\partial^{2} y}{\partial t^{2}}+2 V \frac{\partial^{2} y}{\partial x \partial t}-\left(\frac{F}{m}-V^{2}\right) \frac{\partial^{2} y}{\partial x^{2}}=0
$$

The solution of the equation is found by well-known methods of the quantum theory [12]. To determine the transverse dynamic movements of the caterpillar, the schedule of general movement using normal shapes of oscillations is used. In this case, the solution of equality (2) is:

$$
y(x, t)=\sqrt{\frac{2}{L}} \sum_{k=1}^{\infty} \sin \frac{k \pi x}{L} \cdot\left[c_{1 k} \cos \omega_{k} t+\frac{c_{2 k}}{\omega_{k}} \sin \omega_{k} t\right],
$$


where L - length of the upper part of the caterpillar; $\omega \mathrm{k}$ - frequency of fundamental oscillations in k-shape; $\mathrm{c}_{1 \mathrm{k}}, \mathrm{c}_{2 \mathrm{k}}$ - constants that are determined by the initial conditions.

Fundamental frequency of oscillations of the caterpillar are determined by the formula:

$\omega_{k}=\frac{\pi k}{L} \sqrt{\frac{F}{m}}\left(L-\frac{m}{F} V^{2}\right), \quad k=1,2 \ldots, \quad v_{k}=\frac{\omega_{k}}{2 \pi}$,

Fundamental oscillation frequencies $\omega \mathrm{k}$ depend linearly on the shape of the oscillations $\mathrm{k}$. The coefficient of linear frequency dependence on the number shape is determined by the effort of the tension of the caterpillar $\mathrm{F}$ and its velocity $\mathrm{V}$. When the force $\mathrm{F}$ increases, the frequency of the variations corresponding to different forms increases (Fig. 4).
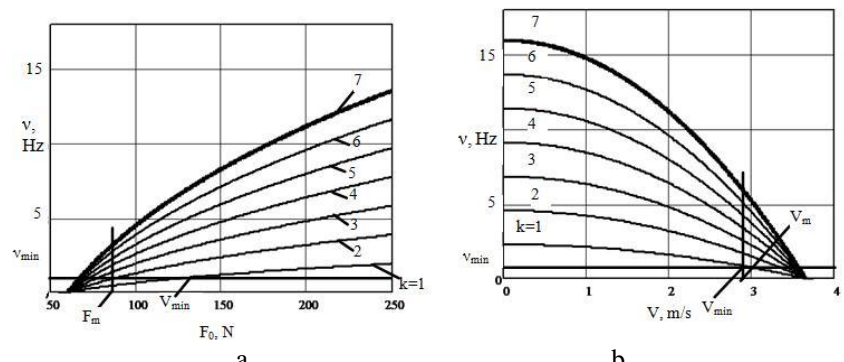

Fig. 4: Dependence of the frequencies of the fundamental values of the caterpillar on different shapes of the tension of the caterpillar (a) and the linear speed of the caterpillar (b).

It follows from the analysis of the graphs that an increase in tension raises the fundamental frequencies of the transverse vibrations of the caterpillar. When reducing the tension of the frequency of oscillations directs to zero and reaches a critical level. Reducing the frequency of oscillations also occurs when increasing the speed of the caterpillar (Fig. 4b). After exceeding some value of the velocity $\mathrm{Vm}$, the frequency of oscillations goes to zero and becomes critical. It is necessary to increase the tension in the upper part of the caterpillar and to reduce the speed of its movement in order to increase the frequency of its fundamental oscillations.

The dependence (3) which determines the transverse movement of the caterpillar includes constant integration which is determined from the initial conditions. Initial conditions may have different character. To establish the laws of the transverse movement of the caterpillar, the initial conditions are given in the shape of an array of constants $\mathrm{c} 1 \mathrm{k}$ and $\mathrm{c} 2 \mathrm{k}$. The constants $\mathrm{c} 1 \mathrm{k}$ correspond to the deviation of the caterpillar from the zero line at time $t=0$. The constants $\mathrm{c} 2 \mathrm{k}$ determine the initial $(\mathrm{at} t=0)$ speeds of the crossings of the caterpillar. The method of successive approximations is a selection of constants that gives a characteristic initial movement of the caterpillar and its velocity. In this case, for the determination of constants, the proposed exponential dependences on the shape of the oscillation number are modulated by trigonometric functions. The dependencies are selected in such a way that at $\mathrm{t}=$ 0 the speed of the crossings of the caterpillar is close to zero, and the movement is maximal. Correspondingly, the constants are calculated according to the formulas:

$$
\begin{aligned}
& c_{1 k}=2 \cos \left(2 \pi k / k_{m}\right) e^{-\frac{k^{2}}{k+1}}, \\
& c_{2 k}=2 \sin \left(2 \pi k / k_{m}\right) e^{-\frac{k^{2}}{k+1}}, \quad k=1,2, \ldots k_{m},
\end{aligned}
$$

where $k_{m}$ - the highest oscillations type number.

Using the formulas (5) we find kinematic initial conditions for the oscillations of the caterpillar by means of its initial transverse movements and the initial speed of the transverse movements of the caterpillar:
$y(0)=\sqrt{\frac{2}{L}} \sum_{k=1}^{\infty} \sin \left(\frac{k \pi x}{L}\right) c_{1 k},\left.\frac{d y}{d t}\right|_{t=0}=\sqrt{\frac{2}{L}} \sum_{k=1}^{\infty} \sin \left(\frac{k \pi x}{L}\right) c_{2 k}$.

The shape of the middle line of the caterpillar at different times is determined according to the initial conditions (6) in formula (3) (Fig. 5a).
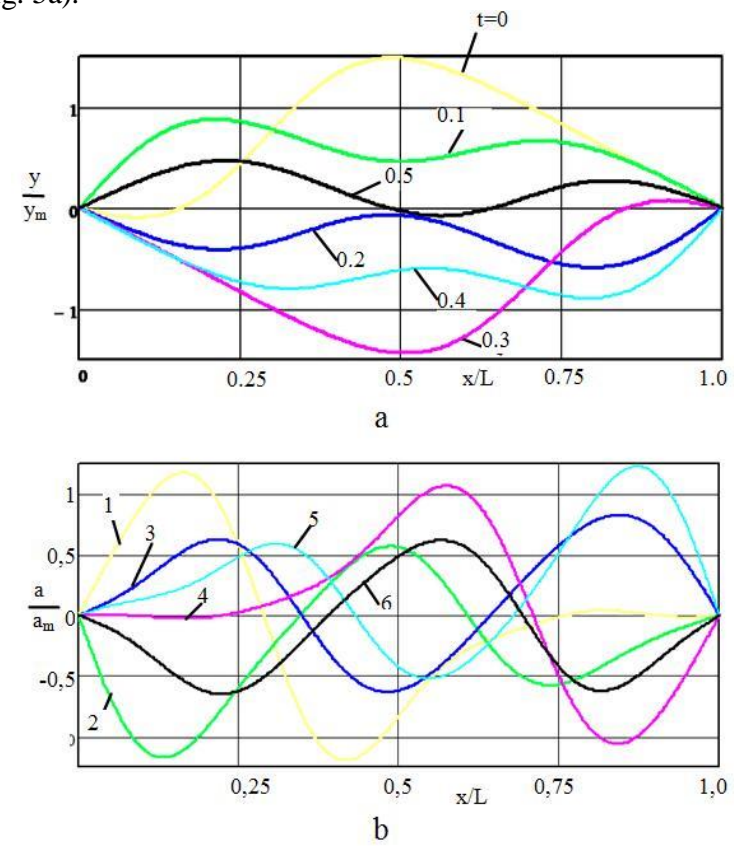

Fig. 5: Changes in the shape of the caterpillar in time due to its oscillations as a system with distributed parameters (a) and acceleration of the crossings of the caterpillar for transverse oscillations (b).

The defined transverse movements of the caterpillar served as the basis for finding the speeds $\mathrm{u}(\mathrm{x}, \mathrm{t})$ of the crossings of the caterpillar and their acceleration a $(x, t)$. Speed and acceleration are found by differentiation of dependence (3). Relatively:

$u(x, t)=\frac{d y(x, t)}{d t}, a(x, t)=\frac{d^{2} y(x, t)}{d t^{2}}$.

The calculated accelerations of the intersections of the caterpillar represent wavy curves similar to the movements (Fig. 5b).

The total transverse inertial load on the caterpillar is determined by the value of the accelerations distributed along the length. The total inertial force is found by calculating the integral:

$$
F_{\Sigma}=m \int_{0}^{L} a(x, t) d x
$$

The calculation by this formula establishes a time-varying law of the change in the total inertial load on the vertical part of the caterpillar (Fig. 6).

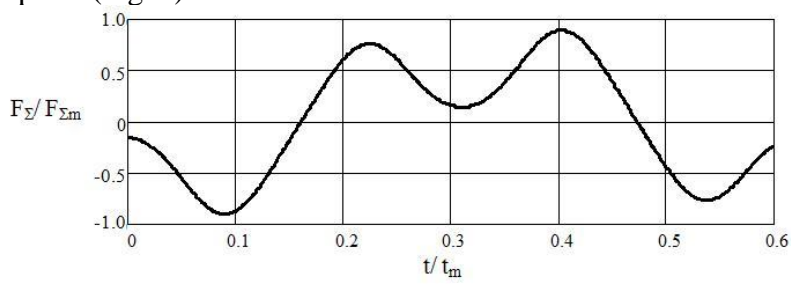

Fig. 6: The inertial load depends on the time that operates on the upper part of the caterpillar at its fundamental oscillation.

The inertial load is changed over time and forms a vertical and dynamic effect $F_{\Sigma} / 2$ on each reel of the caterpillar (see Fig. 3a). 


\section{Mathematical Model of Forced Oscillations of a Caterpillar Mover}

The forced oscillations of the caterpillar arise as a result of the effect of the external dynamic disturbances on the drive of the mover. Disturbances are caused by the appearance of a dynamic time of the tension in the caterpillar. As a result of changing the dynamic tension in the caterpillar, there are no periodic transverse inertial loads that cause the occurrence of transverse oscillations. It was established that transverse discrete loads operate in places of mass concentration on the caterpillar surface and are caused by difference of masses located on different sides from the center line $\mathrm{L}_{0}$ of a flexible caterpillar. Let's consider the causes of transverse discrete loads. When dynamically changing the longitudinal force $F$, the section with thickening acquires the acceleration $a_{x}(t)$ and the inertial forces $F_{1}$ and $F_{2}$ are influenced on the masses $m_{1}$ and $\mathrm{m}_{2}$ located on each side of the caterpillar (Fig. 7).

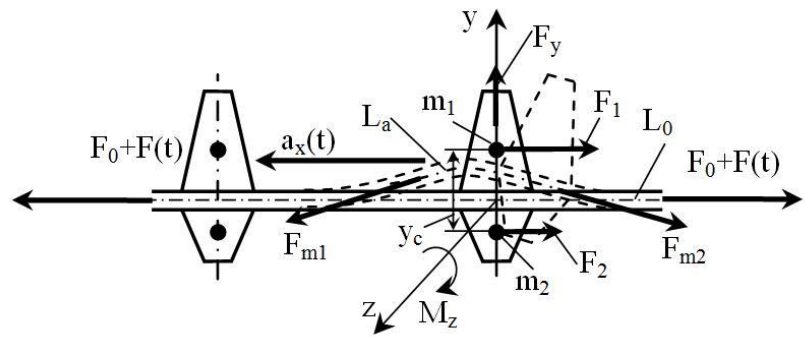

Fig. 7: Scheme of transverse discrete loads on the caterpillar area with thickening (teeth).

Inertia forces $\mathrm{F}_{1}, \mathrm{~F}_{2}$ on both sides of the caterpillar are different and depend on the masses $m_{1}$ and $m_{2}$. To estimate the value of the forces of inertia, we define them as

$\mathrm{F}_{1}=\mathrm{m}_{1} \mathrm{a}, \mathrm{F}_{2}=\mathrm{m}_{2} \mathrm{a}$.

Due to the difference in the forces of inertia there is a torque $\mathrm{M}_{\mathrm{z}}=\mathrm{y}_{\mathrm{c}}\left(\mathrm{F}_{1}-\mathrm{F}_{2}\right)$,

where $y_{c}-$ is the distance between the centers of mass $m_{1}$ and $m_{2}$. The action of the torque leads to the curvature of the elastic line of the caterpillar $\mathrm{L}_{\mathrm{a}}$. At the same time, the tensile forces $\mathrm{Fm}_{1}$ and $\mathrm{Fm}_{2}$ are inclined at acute angles to the center line $\mathrm{L}_{0}$ and are essentially different. Thus, when changing the tension of a caterpillar, there is a transverse force $\mathrm{F}_{\mathrm{y}}$ acting in the direction of the axis $\mathrm{y}$. In the case of changing a discrete tensile force $\mathrm{F}(\mathrm{t})=\mathrm{Fa} \cdot \delta(\mathrm{t})$, the transverse force acting on the caterpillars next to the tooth will also have a discrete origin $\left(c_{y}\right.$ - constant). These loads arise on each caterpillar tooth. Accordingly, a packet of stochastic discrete loads acts in the transverse direction in the case of dynamic changes in a vehicle's caterpillar tension (Fig. 8a).

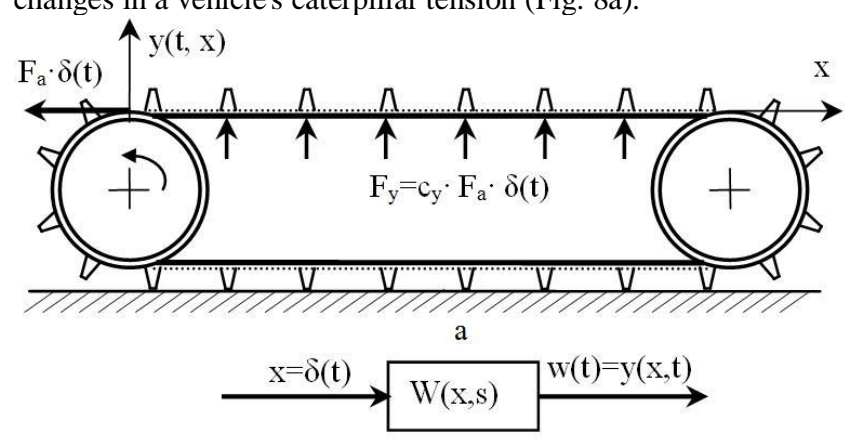

b

Fig. 8: A scheme of discrete loads on a caterpillar (a) and a block- diagram of a dynamic caterpillar system under the action of discrete loading (b).

Initial conditions for oscillations of the caterpillar in speed are formed as a result to the effect of transverse discrete forces, Accordingly, the acquired constant speed:

$\mathrm{c}_{2 \mathrm{~K}} \neq 0, \mathrm{k}=1,2, \ldots$

with constants $\mathrm{c}_{1 \mathrm{~K}}=0$.
Constants are accepted as proportional multipliers with a single discrete function:

$\mathrm{c}_{2 \mathrm{~K}}=\mathrm{c}_{\mathrm{y}} \mathrm{F}_{\mathrm{a}}$.

In this case, the solution of equation (3) will look like:

$y(x, t)=\sqrt{\frac{2}{L}} \sum_{k=1}^{\infty} \sin \left(\frac{k \pi x}{L}\right) \cdot \frac{c_{y} F_{a}}{\omega_{k}} \sin \left(\omega_{k} t\right)$

Movement of the caterpillar according to formula (7) is a consequence of an action on the input parameter system in the form of a longitudinal discrete load of the caterpillar with a single discrete function. In this case, the block-diagram of the dynamic caterpillar system corresponds to the finding of the discrete characteristic of the system W (Fig. 8b). The block contains a transmission function of the system $\mathrm{W}(\mathrm{x}, \mathrm{s})$, which gives the ratio of the Laplace images of the output parameter in the form of movement intersection of the caterpillar which is located at a distance $\mathrm{x}$ from the origin to the Laplace image of the input parameter in the form of dynamic tension of the caterpillar.

According to a block-diagram the transmitting function will correspond to the Laplace transformation of dependence (7). This transformation is found in tables [13] and consists of:

$W(x, s)=\sqrt{\frac{2}{L}} \sum_{k=1}^{\infty} \sin \left(\frac{k \pi x}{L}\right) \cdot \frac{c_{y} F_{a}}{\left(\omega_{k}\right)^{2}} \cdot \frac{1}{T_{k}^{2} S^{2}+1}$.

where $\mathrm{T}_{\mathrm{k}}=1 / \omega_{\mathrm{k}}$.

Let's introduce the designation:

$K_{k}=\sqrt{\frac{2}{L}} \frac{c_{y} F_{a}}{\omega_{k}^{2}} \cdot \sin \left(\frac{k \pi x}{L}\right)$

At the same time, a transmitting function is found in the form of an infinite sum from the formula (8):

$W(s)=\sum_{k=1}^{\infty} \frac{K_{k}}{T_{k}^{2} S^{2}+1}$

The structural diagram corresponding to this transmitting function includes an infinite number of parallel-connected conservative links [14] (Fig. 9a).

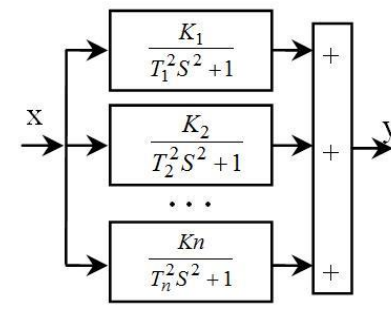

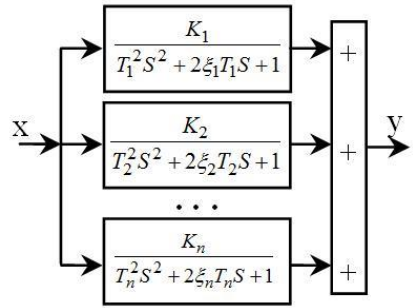

b
Fig. 9: Structural diagram of the caterpillar dynamic system through parallel-connected conservative units (a) and given the structural scheme of the dynamic caterpillar system through the parallel-connected oscillation links (b).

This structure corresponds to a physically unrealistic system because it does not take into account energy losses.

The caterpillar is a massive flexible thread. The energy losses in the dynamic caterpillar system are negligible. It is proposed to take into account the losses by the introduction of equivalent parameters of fading during the replacement of conservative links in the structural scheme into the oscillation links. In this case, the structural scheme of the dynamic system will correspond to an infinite number of parallel- connected oscillation links with corresponding fading parameters $\xi \mathrm{k}$ (Fig. 9b). At $\xi_{k} \rightarrow 0$ this structural scheme will correspond to the connection of conservative links (see fig. 9a) 
Fading parameters are mainly dependent on dissipative processes in the caterpillar material which are insignificant. They could be specified by constant $\xi \mathrm{k}=$ const in the first approximation and refined as a result of a numerical experiment. A mathematical model in the Simulink system has been developed according to the structural scheme. The first seven types of oscillations are taken into account (Fig. 10).

Each block of the model is presented as an oscillation link. Accordingly, a mathematical model is formed in the shape of parallel connected oscillation links. There has been applied a test task when the input parameter of the model is adopted as a step change in stretching load in the caterpillar.

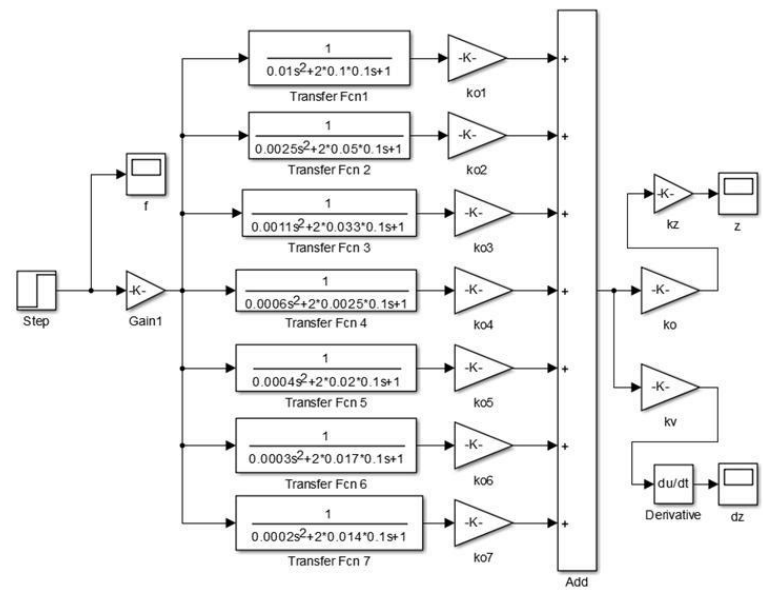

Fig.10: Mathematical model of forced processes in a caterpillar mover that determines the oscillation of the caterpillar as a system with distributed parameters under condition of the first 7 types of fundamental oscillations.

We have the time dependence of the transverse movements of a separate intersection of the caterpillar (average in length of the intersection) and its speed at the output of the model. These parameters are registered with Scope $\mathrm{dz}$ and Scope $\mathrm{z}$ blocks.

Assuming the fact that the parameters of fading of blocks equals zero $\xi 1=\xi 2=\ldots=0$ in the mathematical model, we obtain a mathematical model corresponding to the structural scheme shown in Fig. 9a. This model is used to test its performance and compliance with analytical dependence (3). Confirmation of the results of simulation and the previous calculations are shown in Fig. 5. In order to take into account the energy dissipation values of the fading parameters $\xi 1=\xi 2=\ldots=0,1$ are introduced into the blocks of the mathematical model. It is accepted that they are close to different types of oscillations.

Finally, transition processes of transverse oscillations of the middle intersection of the caterpillar are calculated under condition of the energy loss. The transition function is a smooth fading process (Fig. 11a)

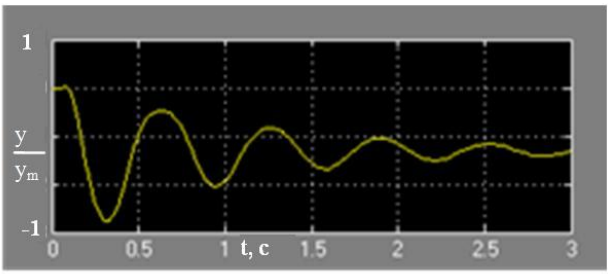

a

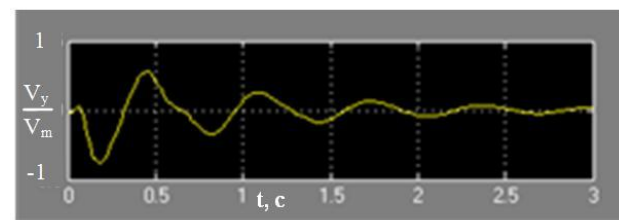

b

Fig. 11: Results of mathematical modelling of transverse movements of the middle intersection of the caterpillar (a) and its speed (b) under the influence of the step change of the tension force.
The transition process is smoothly fading and is generally close to the fading sinusoidal process. On the calculated speed curve (Fig. $11 \mathrm{~b}$ ), nonlinear components could be traced which are especially pronounced in the first period of the oscillation process. They are caused by the influence of higher types of the oscillations of the caterpillar as a system with distributed parameters. These components are important for calculating the high-frequency oscillation processes occurring under random loads of the caterpillar.

The developed mathematical model is used for modeling of transverse oscillations of a caterpillar under different modes of vehicle movement. The modeling of the wheelbase of a caterpillar during acceleration of the vehicle was carried out. The linear law of changing the force in the caterpillar at acceleration is adopted.

Dynamic movements of caterpillar intersection are calculated according to this model. They have the appearance of a fading oscillation process close to that shown in Fig. 11a.

\section{Mathematical Modeling of Oscillation Pro- cesses in a Caterpillar Mover under the Action of Random Loads}

Discrete loads influence on a caterpillar caused by the interaction of the reel projections with the holes in the caterpillar during a specialized vehicle movement. Interaction of projections with holes has a periodic nature but the effort for interaction is changing randomly due to different sizes and location of coupling elements. Thus, the caterpillar is influenced by the longitudinal periodic discrete load which is consistent with the constant effort of the caterpillar tension. The mathematical modeling of the caterpillar oscillations caused by stochastic discrete loads was carried out [15]. A model of random discrete loads has been developed (Fig. 12a) and the calculations of the implementation of the random loads have been performed for this purpose (Fig. 12b).
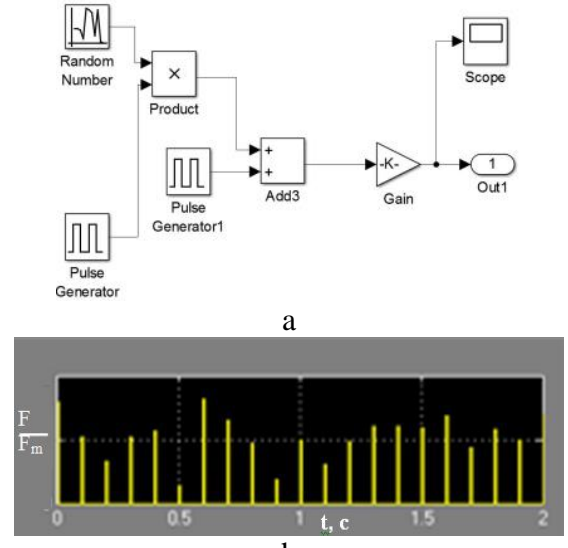

b

Fig. 12: Mathematical model (a) of random discrete loads in a caterpillar mover and calculated implementation of discrete loads (b).

This model is combined with the model of caterpillar dynamics (see Fig. 10). In this case, the transverse movement of the middle intersection of the caterpillar and the speed of its movement under the influence of discrete loads are determined. Transverse movements under the influence of discrete loads have the form of polyharmonic processes (Fig. 13a).

The results of mathematical modeling indicate that the effect of discrete loads causes the transverse oscillations of the caterpillar, which tracks the oscillations in the first and second main forms. The oscillation process has quasi-stationary character with small random variations of the amplitude values. Maxima and minima of co-livings of significant amplitude are observed.

The occurrence of high-frequency changes in speed were found as the result of the simulation (Fig. 13b). 


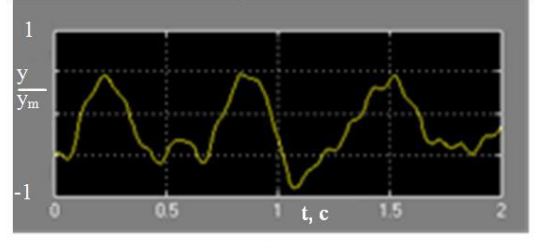

a

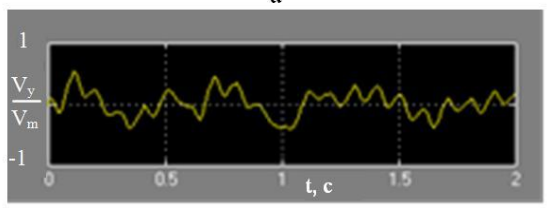

b

Fig.13: Results of mathematical modeling of transverse vibrations of a caterpillar under the action of a random longitudinal discrete load: a movement of the caterpillar intersection; $b$ - the speed of the intersection movements.

Random discrete loads caused by the interaction of the teeth of the driving reel with the caterpillar are the main random loads components. Additional components are random loads in drives and loads caused by the interaction of a caterpillar with a roadway. They operate in the field of low and medium frequencies. For their simulation it is assumed that random loads have a uniform frequency spectrum. Accordingly, they are described by a random process as a "white" noise operating in a limited range of frequencies. The process is generated by a special input block of the model in mathematical modeling. Fadeless transverse movements of the caterpillar occur under the influence of broadband low frequency random loads (Fig. 14a).

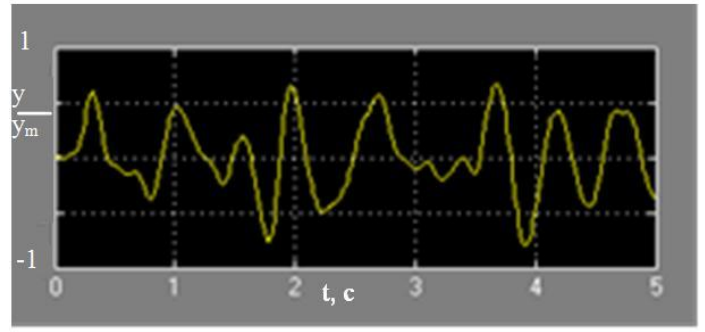

a

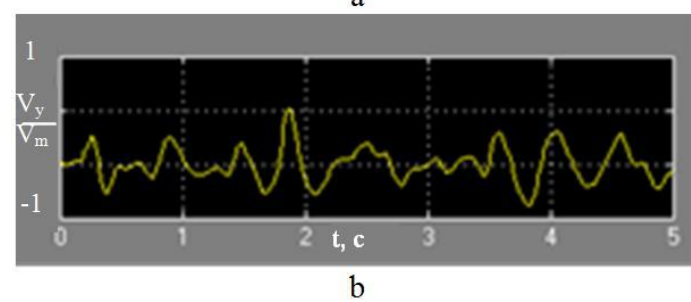

Fig. 14: Results of mathematical modeling of dynamic movements of the caterpillar under the influence of broadband low-frequency random longitudinal loading (a) and the corresponding values of vibration velocity of the intersection of a caterpillar (b).

The oscillations of the caterpillar intersection have the appearance of a polyharmonic process which tracks the first (basic) type of oscillation. The components corresponding to the second type of oscillations are also traced on the curve of caterpillar movements. They are more pronounced in the random process of changing the velocity of transverse oscillations of the caterpillar (Fig. 14b). Oscillations corresponding to those of higher than the second numbers could not be traced on the realizations of random movements and the vibration velocities shown in Fig. 14. This is due to the lack of high-frequency components of random input loads.

There are high-frequency random loads in the drives of the caterpillar and also in the nodes of the specialized vehicle dynamic system. Their simulation has been carried out with the help of the above mentioned model with the appropriate adjustment of the input block parameters [15]. At the same time random highfrequency input parameter as a longitudinal loading of the caterpillar was fed to the input of the dynamic system. He looked like a high-frequency broadband random process (Fig. 15).

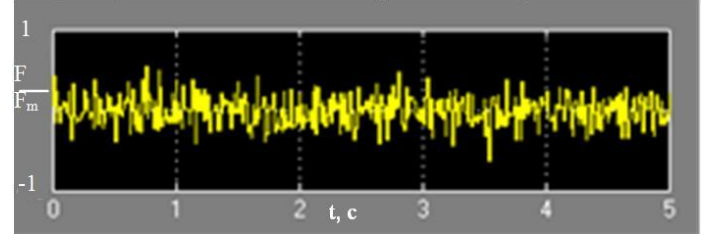

Fig. 15: Broadband random process with essential high-frequency components which was fed to the input of a mathematical model of the system.

Transverse movements of the caterpillar intersections, on which the first, second and higher types of oscillations could be traced, are under the action of a load with high-frequency components (Fig. 16a).
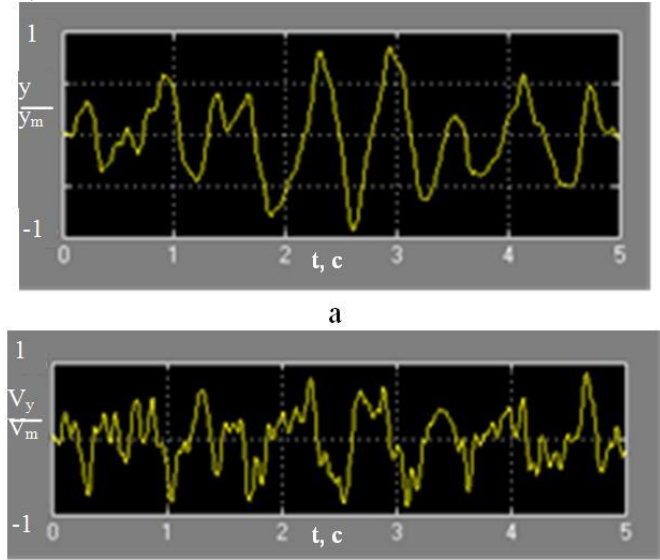

6

Fig. 16: Results of mathematical modeling of transverse movements of the caterpillar intersection(a) and its velocity (b) under the action of longitudinal broadband random load with high frequency components.

High frequency loads generate vibration movements and vibration velocities, which are close to the values of forced oscillations by amplitude values under the influence of low-frequency loads. Frequency spectrum of vibration movements and especially vibration velocities acquires essential high-frequency components. The components of the corresponding types of oscillation up to the seventh one inclusively are traced in the time dependencies of the vibration velocity (Fig. 16b). It causes significant high-frequency oscillations of the caterpillar which reduces its durability. It follows that the occurrence of high-frequency components is an undesirable phenomenon.

It is necessary to implement constructive measures or use damping devices to reduce the negative effects of high frequency oscillation of the caterpillar.

\section{Conclusions}

1. The distribution of parameters (mass) along the length of the specialized vehicle caterpillar causes its own oscillations of the caterpillar as a heavy flexible thread occurring with frequencies corresponding to the first (up to seventh inclusively) types of oscillation. The frequencies are increased with increasing tension in the caterpillar and decreases with increasing speed of the specialized vehicle and the patterns of oscillation of the caterpillar correspond to sinusoidal dependencies.

2. Dynamic discrete changes of the longitudinal load in the caterpillar cause the dynamic transverse loads situated in the areas of the teeth location of the caterpillar resulting in intense forced transverse oscillations of the caterpillar.

3. Discrete longitudinal loads in caterpillar mover occur randomly. In this case, there are transverse oscillations of the caterpillar in which harmonics are traced corresponding to fundamental oscillation frequencies. Components on the first and second patterns within low-frequency disturbances in oscillations are identified, while higher-frequency perturbations show higher patterns of oscillations up to the seventh pattern inclusively. 
4. Increasing the speed of the specialized vehicle results in intense oscillation processes in the caterpillar mover. It is recommended to install inertial dampers in the teeth of the caterpillar and design measures to reduce the dynamic loads in order to reduce the intensity of oscillations.

\section{References}

[1] Kucherov D.P. Prospects for the development of robotic military systems / D.P.Kucherov, Z.M.Kopilova, Yu.V.Myukukhin // Systems of armament and military equipment. - 2007. - Issue. 1 (9) - p. 44-46.

[2] Pat. 79061, IPC F41H 11/16, B62D 57/00. Mobile robotic complex of engineering intelligence and mine clearance / O.M.Guslyakov, V.I.Rudakov, M.I.Vaskivsky and others. // No. 201211644; statements. 10/09/2012; has published Apr 10, 2013, Bul. No. 7 - 10 p.: il. 4.

[3] Strutynsky V.B. Determination of development grounds and characteristics of mobile multi-coordinate robotic machines for materials machining in field conditions /V.B.Strutynsky, A.A.Hurzhi, O.V Kolot, V.E.Polunichev // Scientific Bulletin of the National Mining University. - Dnepr, 2016. - №5 (155). - p.43-51.

[4] Rybak L., Gaponenko E., Chichvarin A., Strutinsky V., Sidorenko R. Computer-Aided Modeling of Dynamics of Manipulator-Tripod with Six Degree of Freedom / World Applied Sciences Journal. N25(2). - 2013. - P.341-346.

[5] Hvorost AG Load of elements of power transfer of a caterpillar tractor at turns / AGKhvorost / / Automobile transport. - 2010 - Issue. 26 - p. 47-52.

[6] Duschenko V.V. Estimation of the effect of the caterpillar machine sweep system on the load of its power plant and transmission / V.V. Dushchenko, I.V. Mysnitskaya // Mechanics and machine building. - Kharkiv: NTU "KhPI". - 2011. - №1. - p. 98102.

[7] Volosikov S.A. The method of determining the critical speed of the caterpillar of the platform by drift / S.A.Volosnikov // Mechanics and machine building. - Kharkiv: NTU "KhPI". - 2016 - №1. - p. 36-44.

[8] Tanchenko A.Yu. On the question of modeling varying in time and load displaced in space / A.Yu.Tanchenko, O.Veretelnik // Mechanics and machine building. - Kharkiv: NTU "KhPI". - 2014 - \# 1. -p. 24-28.

[9] Alexandrov E. E. Fluctuations in transport vehicles / E.E. Alexandrov, Ya.V. Grita, VV Dushchenko et al .: Monograph. - Kharkiv: KPPU, 1996. - $256 \mathrm{p}$.

[10] Vorontsov S.N.Modeling of the effect of the unit perturbing force on the oscillations of the pressed part of the casing body of the caterpillar machine / S.N. Vorontsov, N.S. Yarmak // Mechanics and machine building. - Kharkiv: NTU "KhPI". - 2017 - №1. - p. 274 278.

[11] Koynash V.A. Modeling of caterpillar running gear of excavation machine / V.A. Koynash, V.G. Scarce // Mechanical Engineering and Machine Building. Kharkiv: NTU "KhPI". - 2012. - No. 1. - p. 16-24.

[12] Tymoshenko S.P. Fluctuations in engineering. Tr. from English L.G.Korneichuk / S.P.Timoshenko, D.H. Yang, U.Uiver-M .: Mechanical Engineering, 1985.-472 p.

[13] Oborsky G.A. Modeling of systems / G. A. Oborsky, A. F. Dashchenko, A. V. Usov, D. V. Dmitrishin. - Odessa: Astroprint, 2013. $664 \mathrm{p}$.

[14] Besekersky V.A. The theory of automatic control systems / V.A Besekersky, E.P. Popov. St. Petersburg: Publishing House "Professiya", 2003. - $752 \mathrm{p}$.

[15] Strutynskyi S.V. Definition of vibro displacements of drive systems with laser triangulation meters and setting their integral characteristics via hyper-spectral analysis methods / S.V.Strutynskyi, A.A.Hurzhii // Scientific Bulletin of the National Mining University. - Dnepr, 2017. - №1 . - p.43-51. 